\title{
Use of Mineralocorticoid Receptor Antagonists in Real-world Patients with Heart Failure and Reduced Ejection Fraction: HIJ-HF II study
}

\author{
Atsushi Suzuki, Yuko Matsui, Kimiko Nagara, Tsuyoshi Shiga, Nobuhisa Hagiwara \\ Cardiology, Tokyo Women's Medical University, Japan
}

Mineralocorticoid receptor antagonists (MRAs) improve outcomes in heart failure with reduced ejection fraction (HFrEF), but may be under-utilized in contemporary clinical practice. The aim of this study was to assess predictors of MRAs non-use in HFrEF patients. We studied $331 \mathrm{HFrEF}$ patients $(\mathrm{EF}<40 \%)$ from a multicenter hospital-based cohort consisted of hospitalized HF patients between 2013 and 2014. 217 patients (66\%) were receiving MRAs. There was a significant lower rates of MRAs non-use in patients with ischemic heart disease, without implantable cardioverter defibrillator (ICD) receiving, BNP less than $500 \mathrm{pg} / \mathrm{ml}$ and without angiotensin-converting-enzyme inhibitor (ACE-I) or angiotensin II receptor blocker (ARB). MRAs remain underused in HFrEF patients (Figure). We should understand the indications and benefits of MRAs in appropriate HFrEF patients suggested in guideline. 